Les statuts de la rédaction du Bulletin des médecins suisses (BMS) précisent que l'organe officiel de la FMH doit exprimer, de manière claire et transparente, sa position sur des thèmes importants concernant le corps médical et le système de santé. La promotion d'un libre débat, au sein du corps médical mais également entre les divers acteurs du secteur de la santé, constitue égale- ment une fonction importante du BMS inscrite aux statuts de la rédaction. C'est dans cet esprit que nous publions le manifeste ci-après. Nous remercions les responsables du département DRG de la FMH pour leurs commentaires basés sur la vision de l'organisation.

La rédaction

\title{
Manifeste en faveur d'une sortie des DRG tant qu'il est encore temps
}

\author{
Commentaire de la FMH \\ à la page 1474 .
}

\section{Tenants et aboutissants du manifeste}

A la suite de «l'helvétisation» des DRG (Diagnosis Related Groups, forfaits par cas liés au diagnostic), il apparaît de façon toujours plus nette que les DRG ne permettront pas de réaliser les objectifs fixés en matière de politique de santé, à savoir la transparence, la comparabilité et donc les économies de coûts. Même si l'adaptation à la situation suisse et l'exploitation des enseignements tirés des expériences faites dans d'autres pays semblent judicieuses, la «logique» des DRG orientée sur des données économiques superficielles et de court terme mène sur une mauvaise voie. Selon les spécialistes, les dommages collatéraux pris en compte sous l'appellation globale de «changement de paradigmes» sont toutefois considérables et, de plus, irréversibles. La recherche concomitante mise en place de matière précoce en Suisse ne changera rien à cette situation.

Les réserves émises par les responsables DRG de la FMH sont elles aussi toujours plus vives [1]. Les associations du personnel ont déjà lancé une pétition DRG appelant le Conseil fédéral à prendre des «mesures de soutien» d'ici fin 2009 afin de protéger les patientes et les patients des conséquences négatives des DRG.

Lors du symposium «Economisation de la médecine? - L'introduction des DRG dans les hôpitaux suisses - un défi éthique», organisé par la Commission nationale d'éthique dans le domaine de la médecine humaine (CNE) et l'Académie Suisse des Sciences Médicales (ASSM) le 10 juin 2009 à Berne, il est apparu, à la surprise générale, que l'opinion selon laquelle il faut rendre «pensable l'impensable» et envisager une sortie des DRG tant qu'il est encore temps est largement répandue. Et ce bien que tant les partisans que les opposants des DRG se basent volontiers sur la recherche concomitante, les premiers pour des raisons de légitimation et les seconds en vue d'un apaisement.

Le manifeste a pour objectif de donner un signe. Il n'est pas trop tard pour concevoir, en Suisse, le mandat parlementaire d'établir une structure tarifaire nationale unitaire liée aux prestations de manière à éviter de mettre en jeu le système de santé humainement et professionnellement de grande qualité, en place dans notre pays. Des entretiens avec des parlementaires ont révélé une relative absence de conscience des conséquences des DRG lors de l'acceptation du modèle de financement des hôpitaux fin 2007. Seuls 90 votants se sont exprimés en faveur de ce modèle contre 52 Non et 56 abstentions. Le résultat de la votation parle de lui-même. Par leur «manifeste», les signataires espèrent ramener le débat à un niveau fondamental avant qu'il ne soit trop tard.

\section{Le manifeste: les objectifs de politique de la santé ne peuvent pas être réalisés, les dommages collatéraux pour la médecine sont irréversibles}

Selon l'enquête de l'Académie Suisse des Sciences Médicales (ASSM), nos patients considèrent les soins et l'humanité comme les éléments essentiels de la médecine du XXI ${ }^{\mathrm{e}}$ siècle [2]. Les DRG (Diagnosis Related Groups, forfaits par cas liés au diagnostic) sont diamétralement opposés à cette exigence.

- Sur la base des expériences réalisées depuis dix ans en Australie [3], six ans en Allemagne [4] et trois ans en Suisse [5],

- dans l'intérêt d'un maintien de postes de travail et de formation attrayants pour les médecins, le personnel soignant et d'autres professionnels de la santé,

- dans l'objectif de conserver une médecine professionnelle orientée vers les patients et capable de prendre en compte également les dimensions psychosociales,

les signataires appellent à une sortie des DRG tant qu'il est encore temps.

1. Les DRG ne permettent pas de réaliser l'objectif de politique de santé qui leur a été fixé. Ils ne permettent ni d'économiser des coûts, ni d'améliorer la trans- 
parence et la comparabilité des approvisionnements. Au contraire, les DRG génèrent, de par leur système même, une hausse des coûts administratifs liés au codage, aux contrôles et aux corrections. La saisie toujours plus importante de données des patients favorise par ailleurs des «patients transparents».

2. Par la priorité marquée qu'ils accordent à la rentabilité, les DRG génèrent également une perte inquiétante de professionnalisme pour les professions médicales. Le travail médical devient source de frustrations et de sollicitations excessives pour les médecins de tous niveaux. La pression inappropriée à l'efficience est particulièrement forte dans le domaine des soins. En Allemagne, 30000 emplois à plein temps ont ainsi été perdus suite à l'introduction des DRG.

3. Les DRG sont synonymes de rationnement caché dans le système de santé. Sous couvert de rationalisation, on introduit un rationnement drastique du budget temps. Aujourd'hui déjà pourtant, les patients se plaignent le plus souvent du manque de temps du personnel de soin et des médecins.

4. Avec leur logique pathogène, les DRG rendent les patients plus malades qu'ils ne sont. Par le biais d'un renforcement des barrières entre les processus, axé sur la rentabilité, les DRG sont à l'origine d'un certain mépris des aspects psychosociaux et préventifs. Ils s'opposent donc aux efforts visant à une médecine individuelle axée sur les personnes.
Même si la recherche concomitante prévue est souhaitable en Suisse, elle sera peu performante compte tenu du manque de compétences politiques. En Allemagne, il faut entre trois et quatre ans pour que les mesures de correction portent effet. De plus, la recherche concomitante devrait constater dès à présent, de manière prioritaire, que les DRG ne conviennent pas pour atteindre ses propres objectifs. Il n'est pas trop tard pour concevoir, en Suisse, le mandat parlementaire d'établir une structure tarifaire nationale unitaire liée aux prestations de manière à éviter de mettre en jeu le système de santé humainement et professionnellement de grande qualité, en place dans notre pays.

\section{Références}

1 Swiss DRG - satisfaisant pour les politiques? Eidtorial Bull Méd Suisses. 2009;90:30/31:1147.

2 Stauffacher W, Bircher J (éd). Zukunft Medizin Schweiz. Bâle: EMH Editions médicales suisses; 2002. p. 181-235.

3 Haas NA. Fallpauschalen: Die australische Realität. Deutsches Ärzteblatt 2006;103(25): A1729-30.

4 Rau F, Roeder P, Hensen N (éd). Auswirkungen der DRG-Einführung in Deutschland - Standortbestimmung und Perspektiven. Stuttgart: Kohlhammer; 2009.

5 Commission nationale d'éthique dans le domaine de la médecine humaine NEK/CNE / Académie Suisse des Sciences Médicales ASSM. Economisation de la médecine? - L'introduction des DRG dans les hôpitaux suisses - un défi éthique. Symposium du 10 juin 2009, hôpital de l'Ile, Berne.

\section{Signataires par ordre alphabétique:}

Hansueli Albonico, Dr méd., médecin-chef Médecine complémentaire interdisciplinaire, Hôpital régional, d'Emmental Christoph Cottier, PD Dr méd., ancien Médecin-chef Médecine, Berthoud

Christian Hess, Dr méd., médecin-chef Médecine Hôpital d'Affoltern

Annina Hess-Cabalzar, MA, Psychothérapeute SPV/ASP, présidente de akademie affoltern - menschenmedizin.ch Benedikt Horn, Prof. Dr méd., médecin de famille et chargé de cours de médecine générale, Université de Berne Theodor Itten, lic. phil., président Association Suisse des Psychothérapeutes ASP

Margrit Kessler, présidente Fondation OSP Organisation suisse des patients

Roland Kunz, Dr méd., médecin-chef Gériatrie/Palliative Care, Hôpital d'Affoltern; coprésident palliative.ch

Nadja Lindenmann, Dr méd., co-médecin-chef Médecine Hôpital d'Affoltern

Hans Neuenschwander, Dr méd., médecin principal Palliative Care Programme Ticino

Georg Mang, Dr méd., médecin-chef Médecine Hôpital de Bülach

Robert Maurer, Prof. Dr méd., Illnau

Ueli Münch, Dr méd., ancien médecin-chef Médecine Hôpital de Bülach

Gerhard Rogler, Prof. Dr méd. Dr phil., médecin principal Gastroentérologie Hôpital universitaire de Zurich Anna Sax, Economiste dans le domaine de la santé

Reto Stocker, Prof. Dr méd., service de médecine intensive chirurgicale Hôpital universitaire de Zurich

Dominik Straumann, Prof. Dr méd., Zurich

Urs Strebel, Dr méd., ancien médecin-chef Médecine Hôpital de Männedorf

Erhard Taverna, Dr méd., Urnäsch

Karin Thomas, experte en soins infirmiers BSCN, Centre hospitalier Bienne, présidente de la commission d'association Système de santé de la ssp

Paul Vogt, Prof. Dr méd., chirurgien du cœur, Klinik im Park, Zurich

Erika Ziltener, Patientenstelle Zurich 